\title{
A Direct Variational Approach to Skyrme's Model for Meson Fields
}

\author{
M. J. Esteban
}

Université Pierre et Marie Curie, Laboratoire d'Analyse Numérique (U.A. 189), Tour 55-65-5ème étage, 4, place Jussieu F-75252 Paris Cedex 05, France

\begin{abstract}
The solutions of Skyrme's variational problem describe the structure of mesons in a field of weak energy. The problem consists in minimizing the corresponding energy among the functions from $\mathbb{R}^{3}$ to $S^{3}$ which have a fixed "degree" without making any symmetry assumptions. We prove the existence of minima and study their properties.
\end{abstract}

\section{Introduction}

Let $\phi$ be a function from $\mathbb{R}^{3}$ to $\mathbb{R}^{4}$ such that $\phi\left(\mathbb{R}^{3}\right)$ is contained in the unit sphere of $\mathbb{R}^{4}$. If we write

$$
|\nabla \phi|^{2} \equiv \sum_{i=1}^{4}\left|\nabla \phi_{i}\right|^{2} \quad \text { and } \quad|A(\phi)|^{2}=\sum_{\alpha, \beta=1}^{3}\left|\frac{\partial \phi}{\partial x_{\alpha}} \wedge \frac{\partial \phi}{\partial x_{\beta}}\right|^{2}
$$

(by $a \wedge b$ we denote the alternating exterior product of $a, b \in \mathbb{R}^{4}$ ), we will define the energy associated with the fields $\left\{\phi_{i}\right\}$ by:

$$
\mathscr{E}(\phi)=\frac{\gamma}{4 \pi^{2}} \int_{\mathbb{R}^{3}}\left(\kappa^{2}|\nabla \phi|^{2}+|A(\phi)|^{2}\right) d x,
$$

where $\gamma$ and $\kappa$ are two positive physical constants.

Physical Motivation. T. H. R. Skyrme introduced in [S3] the problem of looking for critical points of the functional $\mathscr{E}$ in an attempt to find a model for the static configurations of a field of mesons.

Recent works seem to indicate that in the large $\mathrm{N}$-limit some gauge field theories $(\mathrm{QCD})$ are equivalent to an effective field theory of mesons (see $[W]$ ). Unfortunately, little is known about the large $N$ limit. Some authors have suggested the study of the case $N=3$, hoping that this case will be very close to the large $N$-limit (see G. t'Hooft [t'H 1,2]).

Another possibility is to attempt simplified field theories as was proposed by Skyrme. Skyrme's idea consisted in adding to the nonlinear $\sigma$-model 
(i.e., $\left.\int_{\mathbb{R}^{3}}|\nabla \phi|^{2} d x\right)$ another term to prevent the solitons in the theory to collapse at certain isolated points with finite energy. The term he chose was $\int_{\mathbb{R}^{3}}|A(\phi)|^{2} d x$, i.e. a term of order 4 in the first order derivatives. Besides he introduced some physical constants, $\kappa$ and $\gamma$ (which are not yet well determined) to fit the experimental data. Recent works (see $[\mathrm{A}, \mathrm{V}, \mathrm{W}]$ ) have shown that Skyrme's choice was more than reasonable, since the theoretical results obtained from his theory fit the experimental measures very well.

For all these reasons Skyrme's model is very useful. However, it takes only into account the pions and omits the other mesons, their masses and their interaction.

The Concrete Mathematical Problem. In Skyrme's model one can define a quantity which is constant for a particular field of mesons, and it has the following analytical expression

$$
N=\frac{1}{2 \pi^{2}} \int_{\mathbb{R}^{3}} \operatorname{det}(\phi, \nabla \phi) d x .
$$

This quantity is actually the degree of $\phi$ composed with a stereographic projection mapping $S^{3}$ (the unit sphere of $\mathbb{R}^{4}$ ) into $\mathbb{R}^{3}$. It measures the "number of times" that the space $\mathbb{R}^{3}$ is mapped into $S^{3}$ by $\phi$. (For more details about the degree see for example $[N]$.)

Let us now define the functional spaces to be considered. Let $Y$ be the following

$$
Y=\left\{\phi: \mathbb{R}^{3} \rightarrow S^{3} \mid \nabla \phi, A(\phi) \in L^{2}\left(\mathbb{R}^{3}, \mathbb{R}^{4}\right)\right\} .
$$

Now we define in $Y$ an approximation-condition as follows:

(P) $\exists\left\{\phi_{n}\right\} \subset Y \cap C^{1}\left(\mathbb{R}^{3}, S^{3}\right)$ such that

$$
\nabla \phi_{n} \underset{n \rightarrow+\infty}{\longrightarrow} \nabla \phi, A\left(\phi_{n}\right) \underset{n \rightarrow+\infty}{\longrightarrow} A(\phi) \text { in } L^{2}\left(\mathbb{R}^{3}, \mathbb{R}^{4}\right) .
$$

Then the set in which we will minimize $\mathscr{E}$ will be:

$$
X=\{\phi \in Y \mid \phi \text { satisfies condition }(\mathrm{P})\} .
$$

Notice that $X$ and $Y$ may be the same set, but this is still an open problem. Anyway, we will only consider functions $\phi$ in $X$. In particular, for all $\phi$ in $X N$ is an integer since the degree is an integer for all smooth functions from $S^{3}$ into itself and $\phi$ satisfies (P). In the sequel $N$ will be denoted by $d(\phi)$.

In order to obtain critical points of $\mathscr{E}$, we will study the following family of minimization problems:

$$
I_{\mathrm{k}}=\inf \left\{\mathscr{E}(\phi) \mid \phi \in X_{k}\right\}, \quad k \in Z,
$$

where $X_{k}=\{\phi \in X \mid d(\phi)=k\}$. In fact, the sets $X_{k}$ should be the connected componets of $X$ in a convenient topology, but this is still an open problem.

Clearly the global minimum of $\mathscr{E}$ in $X$ is $I_{0}=0$, and it is achieved by and only by the constant functions.

In [S3] Skyrme studied this same problem for $k=1$, but considered only 
functions in $X$ with a particular symmetry. In $[K-L]$ Kapitanky and Ladyzenskaia show that there is a minimum of $\mathscr{E}$ among all the functions of $X$ which have this symmetry and whose degree is 1 . In [E2] we generalize this result to the general case $d(\phi)=k \in Z$ and treat an intermediate problem (with less symmetry assumptions). In [E2] we show also that if we denote by $I_{k}^{*}$ the

where

$$
\inf \{\mathscr{E}(\phi) \mid d(\phi)=k, \phi \text { satisfies }(\mathrm{S})\},
$$

$$
\left\{\begin{array}{l}
\exists \omega: \mathbb{R}^{+} \rightarrow \mathbb{R} \text { such that for all } x \in \mathbb{R}^{3}, \\
\phi(x) \equiv\left(\cos \omega(|x|), \frac{x}{|x|} \sin \omega(|x|)\right)
\end{array}\right.
$$

then, at least for $k \neq 0, \pm 1, I_{k}^{*}>I_{k}$.

In this paper we fully solve the more general problems $\left(I_{ \pm 1}\right)$ and give necessary and sufficient conditions to solve $\left(I_{k}\right)$. To do so we use in a fundamental way an inequality of isoperimetric type that we proved in [E1]. It must be emphasized that we make here an important use of the concentration-compactness method due to P. L. Lions (see $[\mathrm{L} 1,2]$ ).

From a mathematical point of view problem $\left(I_{k}\right)$ is very close to the Yang-Mills problem or to that of harmonic maps (see [B-C, T1, 2, U1, 2]). Indeed, the structures of the energy functionals are not very different since the underlying physical problems are closely related.

Furthermore, we would like to explain how the existence of minima of $\left(I_{ \pm 1}\right)$ is useful for the construction of static configurations of meson fields. Skyrme sought fields $\phi$ of minimal energy that are singular at a certain number of isolated points. The number of these singularities is constant and their dynamical behavior is similar to that of a particle coupled to the gradient of the residual field. In [S3] one may find more complete explanations about the idealization of the particles in Skyrme's model.

This paper is organized as follows. First we state our main results. In the second section we give some technical lemmas and in the next two ones we prove our main results. Finally in Appendices 1 and 2 we present some technicalities used in Sects. III and IV.

Notation. Let us now introduce some of the notation we will use below. By $B_{R}$, $B(y, R)$ we will denote the sets:

$$
\left\{x \in \mathbb{R}^{3}|| x \mid<R\right\} \text { and }\left\{x \in \mathbb{R}^{3}|| x-y \mid<R\right\} .
$$

For any set $A,|A|$ will represent the measure (Lebesgue-measure) of $A$ and if $\mathscr{E}$ is the functional defined in (1), $\mathscr{E}(\phi ; A)$ will denote $\left(\gamma / 4 \pi^{2}\right) \int_{A}\left(\kappa^{2}|\nabla \phi|^{2}+|A(\phi)|^{2}\right) d x$.

Finally, for any $x \in \mathbb{R}^{3}, \delta_{x}$ is the Dirac measure at $x$, and for any $a, b, c \in \mathbb{R}^{4}$, we will denote by $a \wedge b \wedge . c$ (resp. $a \wedge b$ ) the alternating exterior product of $a, b, c$ (respectively $a, b)$, which is an element of $\Lambda^{3}\left(\mathbb{R}^{4}\right) \simeq \mathbb{R}^{4}\left(\right.$ respectively $\Lambda^{2}\left(\mathbb{R}^{4}\right)$ ).

\section{Main Results}

Our main results concerning problem $\left(I_{k}\right)$ are the following: 
Theorem 1. For every minimizing sequence of $I_{1}$ (respectively $\left.I_{-1}\right),\left\{\phi_{n}^{ \pm}\right\}_{n \in \mathbb{N}}$, we can find a subsequence of $\left\{\phi_{n}^{ \pm}\right\}_{n}$, still called $\left\{\phi_{n}^{ \pm}\right\}_{n}$, some $y_{n} \in \mathbb{R}^{3}$ and a function $\phi^{+}$ (respectively $\phi^{-}$) in $X$ such that:

$$
\begin{aligned}
& \nabla \phi_{n}^{ \pm}\left(\cdot-y_{n}\right) \underset{n \rightarrow+\infty}{\longrightarrow} \nabla \phi^{ \pm} \\
& A\left(\phi_{n}^{ \pm}\left(\cdot-y_{n}\right)\right) \underset{n \rightarrow+\infty}{\longrightarrow} A\left(\phi^{ \pm}\right)
\end{aligned}
$$

In other words, $d\left(\phi^{ \pm}\right)=1, \mathscr{E}\left(\phi^{ \pm}\right)=I_{+1}$ and $\phi^{+}$(respectively $\phi^{-}$) is a minimum of $I_{1}$ (respectively $I_{-1}$ ).

In the case $k \neq 0, \pm 1$, we are not able to prove such a precise result, and this is due to the fact that little is known about the value of $I_{k}$ and its variation with respect to $k$.

Theorem 2. Let $k$ be in $\mathbb{Z}-\{0, \pm 1\}$. Then, if for all $l \in \mathbb{Z}-\{0, k\}$ we have:

$$
I_{k}<I_{l}+I_{k-l}
$$

there is at least a minimum of $I_{k}$.

Moreover, $\lim _{k \rightarrow+\infty} I_{k}=+\infty$ and for all $k$ in $\mathbb{Z}$ we have: $I_{k}=I_{|k|}$.

Remark. As we will see below, Theorem 2 still holds if instead of assuming (2) for all $l \in \mathbb{Z}-\{0, k\}$, we assume it only for all $l \in \mathbb{Z}$ with $\sqrt{2}|k|<|l|+|k-l|$.

On the other hand, we conjecture that the sequence $\left\{I_{k}\right\}_{k \in \mathbb{N}}$ is monotone increasing but this question remains still open.

Next we try to explain how the study of $I_{+1}$ is a first step to understand the structure of the static configurations of mesons in Skyrme's model.

Let us fix a finite number of points of $\mathbb{R}^{3}, \bar{x}_{1}, \ldots, \bar{x}_{m}$ and $a_{1}, \ldots, a_{m} \in\{1,-1\}$. Following Skyrme's idea of what a static configuration of mesons is, we will look for a sequence $\left\{\phi_{n}\right\}$ in $X$ such that the $\phi_{n}$ are constant on $\mathbb{R}^{3}$ except for the union of small neighborhoods of $\bar{x}_{i}(1 \leqq i \leqq m)$, where all the $\phi_{n}$ behave "like" a minimum of $I_{a_{i}}$. We will construct such a sequence and we will show that it converges in a weak sense to a measure with constant regular part and whose singular part is concentrated at $\left\{\bar{x}_{1}, \ldots, \bar{x}_{m}\right\}$ and is the idealization of $m$ mesons placed at those points, which are called sources. The precise result is as follows:

Let $\bar{x}_{1}, \ldots, \bar{x}_{m} \in \mathbb{R}^{3}, a_{1}, \ldots, a_{m} \in\{1,-1\}$ and $P \in S^{3}$; there exists a sequence $\left\{\phi_{n}\right\}$ in $X$ satisfying the following conditions:

(i) $d\left(\phi_{n}\right)=\sum_{i=1}^{m} a_{i}$ for $n$ large.

(ii) $\phi_{n} \equiv P$ in $\mathbb{R}^{3}-\bigcup_{i=1} B\left(\bar{x}_{i}, \frac{1}{n}\right)$.

(iii) $\mathscr{E}\left(\phi_{n} ; B\left(\bar{x}_{i}, \frac{1}{n}\right)\right) \simeq \frac{n I_{a_{1}}}{2}$ as $n \rightarrow+\infty$.

(iv) $\phi_{n}\left(B\left(\bar{x}_{i}, \frac{1}{n}\right)\right) \supset S^{3}$ for every $i=1, \ldots, m$ and for $n$ large. 
Moreover, $\left\{\operatorname{det}\left(\phi_{n}, \nabla \phi_{n}\right)\right\}$ converges weakly to $\sum_{i=1}^{m} a_{i} \delta_{\bar{x}_{i}}$, where $\delta_{x}$ denotes the Dirac measure at $x$.

Remark. Note that the meaning of this result is that $S^{3}$ may be covered in a small neighborhood of a finite number of fixed points of $\mathbb{R}^{3}$ (sources), with minimal energy. This energy will increase, nevertheless, as rapidly as the radius of the neighborhood of the sources becomes smaller. The high energy zones around the sources represent the mesons. Far away from the sources, the energy is very small (or even zero).

\section{Auxiliary Results}

Before studying the minimization problems $\left(I_{k}\right)$, we will state a basic result proved in [E1], since it is necessary to treat $\left(I_{k}\right)$. It consists in an isoperimetric inequality relating the energy $\mathscr{E}(\phi)$ to the degree $d(\phi)$.

Proposition 4. There exists a positive constant $C$ such that for any $\phi$ in $X$ we have

$$
|d(\phi)|^{3 / 4} \leqq C\|\nabla \phi\|_{L^{2}\left(\mathbb{R}^{3}\right)}\|A(\phi)\|_{L^{2}\left(\mathbb{R}^{3}\right)},
$$

and as it will be shown in Corollary 12, $C$ is achieved in $X$.

Moreover, it was shown in [E1] that when $\operatorname{Im} \phi \subset S^{3}$ and $d(\phi) \neq 0$, then we can prove an inequality better than (3), namely:

$$
\left(\frac{1}{2 \pi^{2}} \int_{\mathbb{R}^{3}}|\operatorname{det}(\phi, \nabla \phi)| d x\right)^{3 / 4} \leqq\|\nabla \phi\|_{L^{2}}\|A(\phi)\|_{L^{2}} C .
$$

Remark. From (3) we may easily infer an inequality relating $d(\phi)$ to $\mathscr{E}(\phi)$ as follows:

$$
|d(\phi)|^{3 / 4} \leqq \frac{4 C \pi^{2}}{\kappa \gamma}\left(\frac{\kappa \gamma}{4 \pi^{2}}\|\nabla \phi\|_{L^{2}}\|A(\phi)\|_{L^{2}}\right) \leqq \frac{2 \pi^{2} C}{\kappa \gamma} \mathscr{E}(\phi) .
$$

Let us now take $\phi$ in $X$ and consider the dilated functions $\phi^{\lambda}(\cdot) \equiv \phi(\cdot / \lambda), \lambda \in \mathbb{R}^{+}$. Since $\nabla \phi^{\lambda}=(1 / \lambda) \nabla \phi(\cdot / \lambda)$, it follows that $d\left(\phi^{\lambda}\right)=d(\phi)$. Indeed this was expected because $\phi^{\lambda}$ and $\phi$ cover $S^{3}$ at a different "speed" but the same "number of times" and with the same orientation.

Using (1) and the above we obtain

$$
\frac{4 \pi^{2}}{\gamma} \mathscr{E}\left(\phi^{\lambda}\right)=\kappa^{2} \int_{\mathbb{R}^{3}}\left|\nabla \phi^{\lambda}\right|^{2} d x+\int_{\mathbb{R}^{3}}\left|A\left(\phi^{\lambda}\right)\right|^{2} d x=\lambda \kappa^{2} \int_{\mathbb{R}^{3}}|\nabla \phi|^{2} d x+\frac{1}{\lambda} \int_{\mathbb{R}^{3}}|A(\phi)|^{2} d x,
$$

and so, the problems $\left(I_{k}\right)$, trivially invariant by translations in $\mathbb{R}^{3}$, are not invariant under the dilations $\phi \rightarrow \phi^{\lambda}$. In any case, we will see next that equality (6) allows us to obtain some information about the minimizing sequences and the minima for $I_{k}$.

Lemma 5. Let us take $k \in \mathbb{Z}$ and $\left\{\phi_{n}\right\}_{n \in \mathbb{N}}$, a minimizing sequence for $I_{k}$. Then,

$$
\kappa^{2} \lim _{n \rightarrow+\infty} \int_{\mathbb{R}^{3}}\left|\nabla \phi_{n}\right|^{2} d x=\lim _{n \rightarrow+\infty} \int_{\mathbb{R}^{3}}\left|A\left(\phi_{n}\right)\right|^{2} d x .
$$


Moreover, if there is a minimum for $I_{k}, \phi$, then,

$$
\kappa^{2} \int_{\mathbb{R}^{3}}|\nabla \phi|^{2} d x=\int_{\mathbb{R}^{3}}|A(\phi)|^{2} d x .
$$

Proof. Assume that the lemma does not hold. For any $\lambda>0$ we consider the sequence $\left\{\phi_{n}^{\lambda}\right\}_{n \in \mathbb{N}}$. We know that for this sequence

$$
\mathscr{E}\left(\phi_{n}^{\lambda}\right)=\frac{\gamma}{4 \pi^{2}}\left[\lambda \kappa^{2} \int_{\mathbb{R}^{3}}\left|\nabla \phi_{n}\right|^{2} d x+\frac{1}{\lambda} \int_{\mathbb{R}^{3}}\left|A\left(\phi_{n}\right)\right|^{2} d x\right] .
$$

Since $\left\{\phi_{n}\right\}$ is a minimizing sequence and since $d\left(\phi_{n}^{\lambda}\right)=d\left(\phi_{n}\right)$ for all $n$ we find that $\lim _{n \rightarrow+\infty} \mathscr{E}\left(\phi_{n}\right) \leqq \lim _{n \rightarrow+\infty} \mathscr{E}\left(\phi_{n}^{\lambda}\right)$ for all $\lambda>0$. Therefore,

$$
(1-\lambda) \kappa^{2} \lim _{n \rightarrow+\infty} \int_{\mathbb{R}^{3}}\left|\nabla \phi_{n}\right|^{2} d x \leqq \frac{(1-\lambda)}{\lambda} \lim _{n \rightarrow+\infty} \int_{\mathbb{R}^{3}}\left|A\left(\phi_{n}\right)\right|^{2} d x .
$$

Now, $\lim _{n \rightarrow+\infty} \int_{\mathbb{R}^{3}}\left|\nabla \phi_{n}\right|^{2} d x \neq 0$, since otherwise $I_{1}$ would be equal to 0 (we see it taking $\left\{\phi_{n}^{\lambda}\right\}$ as minimizing sequence, with $\lambda$ large). But by Proposition $4, I_{1}>0$. Thus, (7) proves the first part of the lemma. The second statement is proved in the same way.

Remark 6. Whenever we consider a function $\phi$ of $X$, we can decrease its energy (in an optimal way) keeping its degree constant, by re-scaling it as follows

$$
\phi \rightarrow \phi^{\bar{\lambda}} \text {, with } \bar{\lambda}=\frac{\|A(\phi)\|_{L^{2}\left(\mathbb{R}^{3}\right)}}{\kappa\|\nabla \phi\|_{L^{2}\left(\mathbb{R}^{3}\right)}} .
$$

Indeed, by using (5) we see that the optimal dilation is achieved when we make equal the two terms of the energy, and this is done for the dilation coefficient $\bar{\lambda}$. This happens because the degree and the functional $\mathscr{F}(\phi)=\kappa\|\nabla \phi\|_{L^{2}\left(\mathbb{R}^{3}\right)}\|A(\phi)\|_{L^{2}\left(\mathbb{R}^{3}\right)}$ are invariant under the dilations $\phi \rightarrow \phi^{\lambda}$, and the inequality $\mathscr{F}(\phi) \leqq \frac{1}{2} \mathscr{E}(\phi)$ becomes an equality when $\kappa\|\nabla \phi\|_{L^{2}\left(\mathbb{R}^{3}\right)}=\|A(\phi)\|_{L^{2}\left(\mathbb{R}^{3}\right)}$.

Lemma 7. For any $\phi$ in $X$ there is $P \in S^{3}$ such that $\int_{\mathbb{R}^{3}}|\phi(x)-P|^{6} d x<+\infty$, i.e., $\phi$ converges to $P$ at infinity in a weak sense.

Proof. By the Poincaré-Wirtinger inequality we know that

where $P_{R}=\frac{1}{\left|B_{R}\right|} \int_{B_{R}} \phi(x) d x$.

$$
\left(\int_{B_{R}}\left|\phi(x)-P_{R}\right|^{6} d x\right)^{1 / 6} \leqq C\left(\int_{B_{R}}|\nabla \phi|^{2} d x\right)^{1 / 2} \leqq C\left(\int_{\mathbb{B}^{3}}|\nabla \phi|^{2} d x\right)^{1 / 2}<+\infty,
$$

Now, since for all $R, P_{R} \in B_{1}$, which is a bounded set of $\mathbb{R}^{4}$, we may apply Fatou's lemma to infer that

$$
\int_{\mathbb{R}^{3}}|\phi(x)-P|^{6} d x<+\infty
$$

where $P$ is any accumulation point of the set $\left\{P_{R}\right\}_{R>0}$. Moreover, the above inequality cannot hold for two different $P$ 's in $S^{3}$, thus $P=\lim _{R \rightarrow+\infty} P_{R}$ and therefore $P \in S^{3}$. 
We note again that the problems $\left(I_{k}\right)$ are invariant under the group of translations in $\mathbb{R}^{3}$. This allows us to prove the following (which is to be expected in view of the general arguments of P. L. Lions [L1, 2]):

Proposition 8. For all $k, l \in \mathbb{Z}$, we have

$$
I_{k} \leqq I_{l}+I_{k-l} .
$$

Proof. First we fix $\varepsilon>0$ and choose $\phi_{l}, \phi_{k-l}$ in $X$ such that

$$
d\left(\phi_{i}\right)=i, \quad\left|\mathscr{E}\left(\phi_{i}\right)-I_{i}\right| \leqq \varepsilon \quad \text { and } \quad\left\|\phi_{i}-(1,0,0,0)\right\|_{L^{6}\left(\mathbb{R}^{3}\right)}<+\infty
$$

for $i=l, k-l$. Then we choose $R>0$ such that:

$$
\mathscr{E}\left(\phi_{i} ; B_{R}^{C}\right) \leqq \varepsilon, \quad i=\ell, k-\ell .
$$

Then we can use Appendix 2 to cut-off $\phi_{l}$ and $\phi_{k-l}$ and obtain two new functions of $X, \psi_{l}, \psi_{k-l}$ satisfying

$$
\begin{aligned}
& \psi_{i \mid B_{R}}=\phi_{i \mid B_{R}}, \psi_{i} \text { constant in } B_{2 R}^{c}, \\
& \left|\mathscr{E}\left(\psi_{i}\right)-\mathscr{E}\left(\phi_{i}\right)\right| \leqq C \varepsilon, \quad i=l, k-l,
\end{aligned}
$$

where $C$ is a fixed positive constant. Moreover, if we choose $\varepsilon$ small enough, it is obvious that $d\left(\psi_{i}\right)=d\left(\phi_{i}\right)=i$ for $i=l, k-l$, since the degree of any function in $X$ is always an integer and $|\operatorname{det}(\phi(x), \nabla \phi(x))| \leqq|\nabla \phi(x)|^{2}+|A(\phi(x))|^{2}$ for any $x$ in $\mathbb{R}^{3}$ and $\phi$ in $X$.

Finally we define $\phi \in X$ by

$$
\begin{aligned}
\phi & \equiv \psi_{l} \text { in } B_{2 R}, \\
\phi(\cdot) & \equiv \psi_{k-l}(\cdot-M) \text { in } B_{2 R}^{c}, \text { with } M \in \mathbb{R}^{3} \text { and }|M|>4 R .
\end{aligned}
$$

For $\phi$ we have

$$
d(\phi)=k \quad \text { and } \quad\left|\mathscr{E}(\phi)-I_{l}-I_{k-l}\right| \leqq 2(C+1) \varepsilon .
$$

Hence, making $\varepsilon$ go to 0 , we obtain (8).

Lemma 9. For all $k$ in $\mathbb{Z}$, the following holds:

$$
I_{k} \geqq \frac{3 \gamma \kappa}{2 \pi^{2}}\left|S^{3}\right||k|
$$

Proof. For any $\phi$ in $X$ we have:

$$
\begin{aligned}
& \int_{\mathbb{R}^{3}}\left(A(\phi)^{2}+\kappa^{2}|\nabla \phi|^{2}\right) d x \geqq 2 \kappa\|A(\phi)\|_{L^{2}\left(\mathbb{R}^{3}\right)}\|\nabla \phi\|_{L^{2}\left(\mathbb{R}^{3}\right)} \\
& \geqq 6 \kappa \int_{\mathbb{R}^{3}}\left|\frac{\partial \phi}{\partial x_{1}} \wedge \frac{\partial \phi}{\partial x_{2}} \wedge \frac{\partial \phi}{\partial x_{3}}\right| d x \quad(\text { see }[\mathrm{E} 1]) .
\end{aligned}
$$

Furthermore, the integral $\int_{\mathbb{B}^{3}}\left|\frac{\partial \phi}{\partial x_{1}} \wedge \frac{\partial \phi}{\partial x_{2}} \wedge \frac{\partial \phi}{\partial x_{3}}\right| d x$ represents the area covered by $\phi\left(\mathbb{R}^{3}\right)$, and hence it is larger or equal than $|d(\phi)|\left|S^{3}\right|$. Then, for all $k \in \mathbb{Z}^{+}$, $I_{k} \geqq\left(3 \gamma \kappa / 2 \pi^{2}\right)\left|S^{3}\right||k|$.

We finish this section with a technical lemma that will be very useful in the sequel. 
Lemma 10. If $a, b, c$ are three vectors in $\mathbb{R}^{3}$, we have:

$$
|a \wedge b \cdot c| \leqq|a \wedge b|^{1 / 2}|a \wedge c|^{1 / 2}|b \wedge c|^{1 / 2} .
$$

Moreover, for all $B \subset \mathbb{R}^{3}$ and $\phi$ in $X$ we have:

$$
\int_{B}\left|\frac{\partial \phi}{\partial x_{1}} \wedge \frac{\partial \phi}{\partial x_{2}} \wedge \frac{\partial \phi}{\partial x_{3}}\right| d x \leqq|B|^{1 / 4}\left(\int_{B}|A(\phi)|^{2} d x\right)^{3 / 4} \text {. }
$$

Proof. If we denote by $l_{a b}$ the quotient $\frac{|a \wedge b|}{|a||b|}$ and by $h_{a b, c}$ the quotient $\frac{|a \wedge b \cdot c|}{|a \wedge b||c|}$, (9) is equivalent to $h_{a b, c} l_{a b} \leqq l_{a b}^{1 / 2} l_{b c}^{1 / 2} l_{a c}^{1 / 2}$. We observe that for any $a, b \in \mathbb{R}^{3}, l_{a b} \in[0,1]$; hence, $l_{a b} \leqq l_{a b}^{1 / 2}$. Moreover, $h_{a b, c} \leqq l_{b c}$ and $h_{a b, c} \leqq l_{a c}$, since $h_{a b, c}$ represents the sine of the angle formed by $c$ with the plane containing $a$ and $b$, while $l_{b c}$ (respectively $l_{a c}$ ) is equal to the sine of the angle between $b$ and $c$ (respectively $a$ and $c$ ). Therefore (9) is proved.

Next, for any $\phi$ in $X$, we denote by $(\varphi, \xi, \phi)$ its spherical coordinates and we consider for all $x \in \mathbb{R}^{3}$ the vectors

$$
\begin{aligned}
& a=\nabla \varphi(x), \quad b=(\cos \varphi(x)) \nabla \xi(x), \\
& c=(\cos \varphi(x) \cos \xi(x)) \nabla \theta(x) .
\end{aligned}
$$

From (9) we obtain that for all $x$,

$$
\begin{aligned}
& \cos ^{2} \varphi(x) \cos \xi(x)|\nabla \theta(x) \wedge \nabla \xi(x) \cdot \nabla \varphi(x)| \\
& \leqq\left.(\cos \varphi(x))(\cos \xi(x))^{1 / 2}|\nabla \theta(x) \wedge \nabla \xi(x)|^{1 / 2}\right) \\
&\left.\cdot(\cos \varphi(x) \cos \xi(x))^{1 / 2}|\nabla \theta(x) \wedge \nabla \varphi(x)|^{1 / 2}\right) \\
&\left.\cdot(\cos \varphi(x))^{1 / 2}|\nabla \xi(x) \wedge \nabla \varphi(x)|^{1 / 2}\right),
\end{aligned}
$$

and finally we apply Hölder's inequality to obtain (10) by using the representation of $d(\phi)$ and $A(\phi)$ in terms of the spherical coordinates given in Appendix 1.

\section{Proof of Theorem 1}

In this section we will just consider the case $k=1$. As we have pointed out above, the case $k=-1$ can be treated in a similar way.

Let $\left\{\phi_{n}\right\}_{n \in \mathbb{N}}$ be a minimizing sequence for $I_{1}$. The energies of the $\phi_{n}$ 's will be uniformly bounded and thus, we may extract a subsequence, still denoted by $\left\{\phi_{n}\right\}_{n \in \mathbb{N}}$, such that there is a $\phi$ in $X$ satisfying:

$$
\begin{array}{ll}
\nabla \phi_{n} \underset{n \rightarrow+\infty}{\longrightarrow} \nabla \phi & \text { in } L^{2}\left(\mathbb{R}^{3}, \mathbb{R}^{4}\right) \text {-weak } \\
A\left(\phi_{n}\right) \underset{n \rightarrow+\infty}{\longrightarrow} A(\phi) & \text { in } L^{2}\left(\mathbb{R}^{3}, \mathbb{R}^{4}\right) \text {-weak, } \\
\phi_{n} \underset{n \rightarrow+\infty}{\longrightarrow} \phi & \text { in } L^{\infty}\left(\mathbb{R}^{3}\right) \text {-weak* }
\end{array}
$$

Indeed, the weak limit of $\left\{A\left(\phi_{n}\right)\right\}$ coincides with $A(\phi)$ because of the particular structure of $A$, as it is shown for a large class of nonlinear functions $\mathrm{A}$ in [B (see Theorem 6.2), R, M or TAR]. 
Next we observe that for any function $\phi$ in $X$, we can obtain another function of $X, \tilde{\phi}$ with

$$
d(\phi)=d(\tilde{\phi}), \quad \mathscr{E}(\phi)=\mathscr{E}(\tilde{\phi}), \quad \tilde{\phi}(+\infty)=e=(1,0,0,0),
$$

and we do it simply by rotating the image of $\phi$. If we rotate all the $\phi_{n}$ 's in order that $\phi_{n}(+\infty)=e$, besides (11) we obtain also:

$$
\begin{array}{cc}
\phi_{n}-e \underset{n \rightarrow+\infty}{\longrightarrow} \phi-e & \text { in } L^{6}\left(\mathbb{R}^{3}, \mathbb{R}^{4}\right) \text {-weak, } \\
\phi_{n} \underset{n \rightarrow+\infty}{\longrightarrow} \phi & \text { in } L_{\mathrm{loc}}^{2}\left(\mathbb{R}^{3}, \mathbb{R}^{4}\right),
\end{array}
$$

where possibly $\left\{\phi_{n}\right\}$ is now a subsequence of the original minimizing sequence.

By using the weak lower semicontinuity of the $L^{2}$-norm, we obtain from (11)

$$
\mathscr{E}(\phi) \leqq \frac{\lim }{n \rightarrow+\infty} \mathscr{E}\left(\phi_{n}\right)=I_{1} .
$$

Thus, if we had $d(\phi)=1, \phi$ would be a solution to our problem.

If for all $\phi$ in $X$, we denote by $(\varphi, \xi, \theta)$ its spherical coordinates, as we do in Appendix 1, we see that for all $n$ the function

$$
f_{n}=\delta\left(\cos \varphi_{n} \cos \xi_{n}\right)^{2} \theta_{n}^{6}+\delta\left(\cos \varphi_{n}\right)^{2} \xi_{n}^{6}+\delta \varphi_{n}^{6}+\kappa^{2}\left|\nabla \phi_{n}\right|^{2}+\left|A\left(\phi_{n}\right)\right|^{2}+\delta\left|\phi_{n}-e\right|^{6}
$$

are uniformly bounded in $L^{1}\left(\mathbb{R}^{3}\right)$ for any fixed positive constant $\delta$ (it will be fixed below). Indeed as we see in Appendix $1,\left|\nabla \phi_{n}\right|^{2}$ can be also written as $\cos ^{2} \varphi_{n} \cos ^{2} \xi_{n}\left|\nabla \theta_{n}\right|^{2}+\cos ^{2} \varphi_{n}\left|\nabla \xi_{n}\right|^{2}+\left|\nabla \varphi_{n}\right|^{2}$, and $e$ has been chosen in order to have $\left\|\theta_{n}\right\|_{L^{6}\left(\mathbb{R}^{3}\right)},\left\|\xi_{n}\right\|_{L^{6}\left(\mathbb{R}^{3}\right)},\left\|\varphi_{n}\right\|_{L^{6}\left(\mathbb{R}^{3}\right)}<+\infty$.

Therefore, $\left\|f_{n}\right\|_{L^{1}\left(\mathbb{R}^{3}\right)}=\lambda_{n}$, and $\left\{\lambda_{n}\right\}_{n \in \mathbb{N}}$ is a sequence bounded away from 0 and $+\infty$.

Now we introduce the concentration function of $\phi_{n}$ as follows:

$$
Q_{n}(t)=\sup _{y \in \mathbb{B}^{3}} \int_{B(y, t)} f_{n}(x) d x, \quad t>0 .
$$

The notion of the concentration function of a measure was first introduced by Levy [Le] and has been recently used by P. L. Lions (see [L1,2]) to give a general method (concentration-compactness) for the existence of minima of minimization problems. This so-called concentration-compactness method will be used here to infer the existence of at least one minimum for $I_{1}$.

P. L. Lions proved in $[\mathrm{L} 1,2]$ that for a sequence as $\left\{\phi_{n}\right\}$ (or $\left\{f_{n}\right\}$ ), there are only three possibilities:

(i) (compactness) There exists a sequence $\left\{y_{n}\right\}$ in $\mathbb{R}^{3}$ such that:

$$
\forall \varepsilon>0 \exists R>0 \text { s.t. for all } n, \int_{B\left(v_{n}, R\right)^{c}} f_{n}(x) d x \leqq \varepsilon
$$

(ii) (vanishing) For all $R>0$,

$$
\lim _{n \rightarrow+\infty} \sup _{y \in \mathbb{R}^{3}} \int_{B(y, R)} f_{n}(x) d x=0
$$


(iii) (dichotomy) There exists a sequence $\left\{y_{n}\right\}$ in $\mathbb{R}^{3}$ and two real positive numbers, $a$, $b$, such that $a+b=1$ and

$$
\forall \varepsilon>0, \exists R, R_{n}>0, \lim _{n \rightarrow+\infty} R_{n}=+\infty
$$

such that

$$
\begin{aligned}
& \left|\int_{B\left(y_{n}, R\right)} f_{n}(x) d x-a \lambda_{n}\right| \leqq \varepsilon \\
& \left|\int_{B\left(y_{n}, R_{n}\right)^{c}} f_{n}(x) d x-b \lambda_{n}\right| \leqq \varepsilon
\end{aligned}
$$

where $\lambda_{n}=\left\|f_{n}\right\|_{L^{1}\left(\mathbb{R}^{3}\right)}$.

Let us now prove that (ii) and (iii) are impossible in our case. We will do it in several steps.

Proposition 11. There does not exist $R>0$ such that (17) holds. In other words, the sequence $\left\{\phi_{n}\right\}$ cannot vanish.

Proof. First let us show that if (17) holds, then:

$$
\lim _{n \rightarrow+\infty} \int_{\mathbb{R}^{3}}\left|\phi_{n}-e\right|^{6 \alpha} d x=0 \text { for all } \alpha>1 .
$$

We will do it by using an idea of P. L. Lions in [L1]. We choose a countable set of points of $\mathbb{R}^{3},\left\{y_{i}\right\}$ such that

$$
-\bigcup_{i} B\left(y_{i}, R\right)=\mathbb{R}^{3} \text { and }
$$

—every $x$ in $\mathbb{R}^{3}$ is at most in $m$ of these balls $B\left(y_{i}, R\right)$, where $m$ is a fixed constant.

Then, since by the Sobolev's imbeddings, $W^{1,1}\left(B_{R}\right) \hookrightarrow L^{\alpha}\left(B_{R}\right), \alpha \in[1,3 / 2]$, we may write:

$$
\int_{B\left(y_{i}, R\right)}\left|\phi_{n}-e\right|^{6 \alpha} d x \leqq C\left(\int_{B\left(y_{1}, R\right)}\left(\left|\phi_{n}-e\right|^{6}+6\left|\phi_{n}-e\right|^{5}\left|\nabla \phi_{n}\right|\right) d x\right)^{\alpha}, \forall i .
$$

Next, by using Hölder's inequality we obtain

$$
\begin{aligned}
\int_{B\left(y_{i}, R\right)}\left|\phi_{n}-e\right|^{5}\left|\nabla \phi_{n}\right| d x & \leqq\left(\int_{B\left(y_{i}, R\right)}\left|\phi_{n}-e\right|^{10} d x\right)^{1 / 2}\left(\int_{B\left(y_{i}, R\right)}\left|\nabla \phi_{n}\right|^{2} d x\right)^{1 / 2} \\
& \leqq 4\left(\int_{B\left(y_{i}, R\right)}\left|\phi_{n}-e\right|^{6} d x\right)^{1 / 2}\left(\int_{B\left(y_{i}, R\right)}\left|\nabla \phi_{n}\right|^{2} d x\right)^{1 / 2},
\end{aligned}
$$

where we have used that $|e|=1,\left\|\phi_{n}\right\|_{L^{\infty}\left(\mathbb{R}^{3}\right)} \leqq 1$.

Therefore, by (17),

$$
\left.\sup _{i} \int_{B\left(y_{i}, R\right)}\left|\phi_{n}-e\right|^{6}+6\left|\phi_{n}-e\right|^{5}\left|\nabla \phi_{n}\right|\right) d x=a_{n} \underset{n \rightarrow+\infty}{\longrightarrow} 0
$$

and then, for every $i$,

$$
\int_{B\left(y_{i}, R\right)}\left|\phi_{n}-e\right|^{6 \alpha} d x \leqq C a_{n}^{\alpha-1} \int_{B\left(y_{i}, R\right)}\left(\left|\phi_{n}-e\right|^{6}+\left|\nabla \phi_{n}\right|^{2}\right) d x,
$$


where $C$ is independent of $n$, and $\lim _{n \rightarrow+\infty} a_{n}=0$. Finally we deduce from $(20)$ that

$$
\begin{aligned}
\left\|\phi_{n}-e\right\|_{L^{6 \alpha\left(\mathbb{R}^{3}\right)}}^{6 \alpha} & \leqq C a_{n}^{\alpha-1} m\left(\left\|\phi_{n}-e\right\|_{L^{6}\left(\mathbb{R}^{3}\right)}^{6}+\left\|\nabla \phi_{n}\right\|_{L^{2}\left(\mathbb{R}^{3}\right)}^{2}\right) \\
& \leqq C^{\prime} a_{n}^{\alpha-1} \underset{n \rightarrow+\infty}{\longrightarrow} 0
\end{aligned}
$$

and since $\left\|\phi_{n}\right\|_{L_{\left(\mathbb{R}^{3}\right)}}=1$ for all $n,(19)$ is proved.

Now, for every $n \in \mathbb{N}$ we consider the set

$$
A_{n}=\left\{x \in \mathbb{R}^{3}|| \phi_{n}(x)-e \mid \geqq 1\right\}
$$

From (19) we deduce that $\lim _{n \rightarrow+\infty}\left|A_{n}\right|=0$.

On the other hand, since $d\left(\phi_{n}\right)=1$ for all $n$, we know that $\phi_{n}\left(\mathbb{R}^{3}\right) \supset S^{3}$. Moreover, by the definition of $A_{n}, \phi_{n}\left(A_{n}\right)$ must contain the half-sphere of $S^{3}$ opposite to $e$. Therefore,

$$
\int_{A_{n}}\left|\frac{\partial \phi_{n}}{\partial x_{1}} \wedge \frac{\partial \phi_{n}}{\partial x_{2}} \wedge \frac{\partial \phi_{n}}{\partial x_{3}}\right| d x \geqq \operatorname{area}\left(\phi_{n}\left(A_{n}\right)\right) \geqq \frac{\left|S^{3}\right|}{2}=\pi^{2}
$$

Finally, we observe that we reach a contradiction since by Lemma 10 $\int_{A_{n}}\left|\frac{\partial \phi_{n}}{\partial x_{1}} \wedge \frac{\partial \phi_{n}}{\partial x_{2}} \wedge \frac{\partial \phi_{n}}{\partial x_{3}}\right| d x$ is bounded by a constant times $\left|A_{n}\right| \mathscr{E}\left(\phi_{n}\right)^{3 / 4}$, and this converges to 0 as $n$ goes to $+\infty$.

Let us now see that the only possibility for a minimizing sequence like $\left\{\phi_{n}\right\}$ is the compactness in the sense of (16). To do so we have to exclude first the possibility of dichotomy.

Proposition 12. Under our assumptions, (18) cannot occur. Therefore, (16) holds, i.e., $\left\{f_{n}\left(\cdot-y_{n}\right)\right\}_{n}$ is tight for some $y_{n} \in \mathbb{R}^{3}$.

Proof. Assume that (18) is verified. First, since $R_{n} \rightarrow+\infty$, we may suppose that $8 \leqq 8 R \leqq R_{n}$. Then by using Appendix 2 , we may "cut $\phi_{n}$ into two pieces," that is, we may define two new functions in $X, \phi_{n}^{1}$ and $\phi_{n}^{2}$, which satisfy:

$$
\begin{gathered}
\phi_{n}^{1} \equiv \phi_{n} \text { in } B_{R}, \quad \phi_{n}^{1} \equiv(1,0,0,0) \text { in } B_{8 R}^{c}, \\
\mathscr{E}\left(\phi_{n}^{1} ; B_{8 R}-B_{R}\right) \leqq C \mathscr{E}\left(\phi_{n} ; B_{8 R}-B_{R}\right) \leqq C \varepsilon, \text { and } \\
\phi_{n}^{2} \equiv \phi_{n} \text { in } B_{R_{n}}^{c}, \quad \phi_{n}^{2} \equiv(1,0,0,0) \text { in } B_{R_{n} / 8}, \\
\mathscr{E}\left(\phi_{n}^{2} ; B_{R_{n}}-B_{R_{n} / 8}\right) \leqq C \mathscr{E}\left(\phi_{n}^{2} ; B_{R_{n}}-B_{R_{n} / 8}\right) \leqq C \varepsilon
\end{gathered}
$$

Moreover, if we take $\varepsilon$ small enough, we may deduce from (21)-(22) that

$$
d\left(\phi_{n}^{1}\right)+d\left(\phi_{n}^{2}\right)=1 .
$$

Therefore, since the degree of any fuction in $X$ is an integer, for all $n$ we must have

$$
d\left(\phi_{n}^{1}\right)=k_{n}, \quad d\left(\phi_{n}^{2}\right)=l_{n} ; \quad k_{n}, l_{n} \in \mathbb{Z}, \quad k_{n}+l_{n}=1 .
$$


Now two cases are possible:

$$
\begin{aligned}
& -k_{n}, l_{n} \in\{0,1\} \\
& -\left|k_{n}\right|+\left|l_{n}\right| \geqq 3 .
\end{aligned}
$$

In the first case we would have a sequence in $X,\left\{\psi_{n}\right\}$, with

$$
d\left(\psi_{n}\right)=1, \lim _{n \rightarrow+\infty} \mathscr{E}\left(\psi_{n}\right)<I_{1} .
$$

Indeed the equality $\lim _{n \rightarrow+\infty} \mathscr{E}\left(\psi_{n}\right)=I_{1}$ is incompatible with (18). But (23) can never hold, since it contradicts $I_{1}$ 's definition. Thus the first case is impossible. Let us now see that the same happens in the second one.

Without loss of generality, we assume that $\left|k_{n}\right| \geqq 2$. Then, from $\phi_{n}^{1}$ 's definition we infer that for $n$ large we have

$$
\mathscr{E}\left(\phi_{n}^{1}\right) \leqq C \varepsilon+I_{1}+\delta(L+1) I_{1},
$$

where $L$ is the best constant in the inequality

$$
\int_{\mathbb{R}^{3}}|\phi-e|^{6} d x \leqq L\left(\int_{\mathbb{R}^{3}}|\nabla \phi|^{2} d x\right)^{3} .
$$

Hence, if we choose $\delta$ such that $1+\delta(L+1)<2 / \sqrt{2}$ and $\varepsilon$ small, we find

$$
I_{k_{n}} \leqq \mathscr{E}\left(\phi_{n}^{1}\right)<\frac{2}{\sqrt{2}} I_{1},
$$

and this is enough to conclude, since it contradicts Lemma 9 or (28).

Proposition 13. Let $\left\{\phi_{n}\right\}$ be a sequence in $X$ such that $\left\{\left|\frac{\partial \phi_{n}}{\partial x_{1}} \wedge \frac{\partial \phi_{n}}{\partial x_{2}} \wedge \frac{\partial \phi_{n}}{\partial x_{3}}\right|\left(\cdot-y_{n}\right) \mid\right\}_{n}$ is tight for some $y_{n} \in \mathbb{R}^{3}$, then, there exist a countable set of points, $\bar{x}_{1}, \ldots, \bar{x}_{n}, \ldots$ in $\mathbb{R}^{3}$ and $v_{1}, \ldots, v_{n}, \ldots$ in $\mathbb{R}$ satisfying:

$$
\frac{1}{2 \pi^{2}} \operatorname{det}\left(\phi_{n}, \nabla \phi_{n}\right) \underset{n \rightarrow+\infty}{\longrightarrow} \frac{1}{2 \pi^{2}} \operatorname{det}(\phi, \nabla \phi)+\sum_{i=1}^{m} v_{i} \delta_{\bar{x}_{i}} \text { in } \mathscr{D}^{\prime}\left(\mathbb{R}^{3}\right) \text {-weak, }
$$

where by $\left\{\phi_{n}\right\}$ we denote $\left\{\phi_{n}\left(\cdot+y_{n}\right)\right\}$ and by $\phi$, its weak limit.

Moreover, the sequence of $L^{1}$-functions $\left\{\left|\frac{\partial \phi_{n}}{\partial x_{1}} \wedge \frac{\partial \phi_{n}}{\partial x_{2}} \wedge \frac{\partial \phi_{n}}{\partial x_{3}}\right|\right\}_{n \in \mathbb{N}}$ converges weakly to a measure $\mu$ satisfying

$$
\mu \geqq\left|\frac{\partial \phi}{\partial x_{1}} \wedge \frac{\partial \phi}{\partial x_{2}} \wedge \frac{\partial \phi}{\partial x_{3}}\right|+\sum_{i=1}^{m} \mu_{i} \delta_{\bar{x}_{i}}
$$

with $\mu_{i} \geqq 2 \pi^{2} v_{i}^{3 / 4}$ for every $i=1, \ldots m$.

Proof. The result is proved in quite a similar way as Lemma I.1 in [L2]. In order to do the same here we just have to check some technical details.

First we have to prove that there exists a positive constant $C$ such that if $\phi$ were equal to the north pole of $S^{3}$ almost everywhere we would have

for all $\varphi \in \mathscr{D}^{\prime}\left(\mathbb{R}^{3}\right)$.

$$
\left(\int_{\mathbb{R}^{3}} \varphi^{4} d v\right)^{3 / 4} \leqq C \int_{\mathbb{R}^{3}} \varphi^{3} d \mu,
$$


This is easily verified. By Proposition 4 we have

$$
\left|\int_{\mathbb{R}^{3}} \operatorname{det}\left(\varphi \phi_{n}, \nabla\left(\varphi \phi_{n}\right)\right) d x\right|^{3 / 4} \leqq C_{1} \int_{\mathbb{R}^{3}}\left|\frac{\partial\left(\varphi \phi_{n}\right)}{\partial x_{1}} \wedge \cdots \wedge \frac{\partial\left(\varphi \phi_{n}\right)}{\partial x_{3}}\right| d x
$$

for all $\varphi \in \mathscr{D}^{\prime}\left(\mathbb{R}^{3}\right)$. Since the left-hand part of this inequality is equal to $\mid \int \varphi_{\mathbb{R}^{3}} \varphi^{4} \times$ $\left.\operatorname{det}\left(\phi_{n}, \nabla \phi_{n}\right) d x\right|^{3 / 4},(26)$ will be proved if we show that the right-hand integral converges to $\int_{\mathbb{R}^{3}} \varphi^{3} d \mu$. But,

$$
\begin{aligned}
\int_{\mathbb{R}^{3}}\left|\frac{\partial\left(\varphi \phi_{n}\right)}{\partial x_{1}} \wedge \cdots \wedge \frac{\partial\left(\varphi \phi_{n}\right)}{\partial x_{3}}\right| d x=\int_{\mathbb{R}^{3}}|\varphi|^{3}\left|\frac{\partial \phi_{n}}{\partial x_{1}} \wedge \cdots \wedge \frac{\partial \phi_{n}}{\partial x_{3}}\right| d x \\
+\sum_{(i, j, k) \in Q} \int_{\mathbb{R}^{3}}|\varphi|^{2}\left|\frac{\partial \varphi}{\partial x_{i}}\right| \phi_{n} \wedge \frac{\partial \phi_{n}}{\partial x_{j}} \wedge \frac{\partial \phi_{n}}{\partial x_{k}} \mid d x,
\end{aligned}
$$

where $Q$ is the set of permutations of $(1,2,3)$.

Then, (26) is proved because $\phi_{n} \underset{n \rightarrow+\infty}{\longrightarrow} 0$ in $L_{\text {loc }}^{2}\left(\mathbb{R}^{4}, \mathbb{R}^{4}\right)$ and $A\left(\phi_{n}\right) \underset{n \rightarrow+\infty}{\longrightarrow} 0$ in $L^{2}\left(\mathbb{R}^{3}, \mathbb{R}^{4}\right)$-weak.

To treat the case where $\phi$ is not equal to the north pole almost everywhere we need some additional information about the convergence of the sequence $\left\{\operatorname{det}\left(\phi_{n}, \nabla \phi_{n}\right)\right\}$ towards $v$.

In particular we need to show that

$$
\int_{\mathbb{B}^{3}} \varphi^{4} \operatorname{det}\left(\phi_{n}-\phi, \nabla\left(\phi_{n}-\phi\right)\right) d x-\int_{\mathbb{B}^{3}} \varphi^{4} \operatorname{det}\left(\phi_{n}, \nabla \phi_{n}\right) d x \underset{n \rightarrow+\infty}{\longrightarrow} \int_{\mathbb{R}^{3}} \varphi^{4} \operatorname{det}(\phi, \nabla \phi) d x .
$$

We set $\psi_{n}=\phi_{n}-\phi$ and make the convention that $\partial f / \partial x_{0} \equiv f$ for all $f$. Then we compute easily that

$$
\begin{gathered}
\operatorname{det}\left(\psi_{n}, \nabla \psi_{n}\right)-\operatorname{det}\left(\phi_{n}, \nabla \phi_{n}\right)-\operatorname{det}(\phi, \nabla \phi) \\
=\sum_{(\alpha, \beta, \lambda, \gamma) \in \mathscr{P}}\left[a_{\lambda, \gamma}^{\alpha, \beta} \operatorname{det}\left(\frac{\partial \phi}{\partial x_{\alpha}}, \frac{\partial \phi}{\partial x_{\beta}}, \frac{\partial \phi}{\partial x_{\lambda}}, \frac{\partial \psi_{n}}{\partial x_{\gamma}}\right)\right. \\
+b_{\lambda, \gamma}^{\alpha, \beta} \operatorname{det}\left(\frac{\partial \phi}{\partial x_{\alpha}}, \frac{\partial \phi}{\partial x_{\beta}}, \frac{\partial \psi_{n}}{\partial x_{\lambda}}, \frac{\partial \psi_{n}}{\partial x_{\gamma}}\right) \\
\left.+c_{\lambda, \gamma}^{\alpha, \beta} \operatorname{det}\left(\frac{\partial \phi}{\partial x_{\alpha}}, \frac{\partial \psi_{n}}{\partial x_{\beta}}, \frac{\partial \psi_{n}}{\partial x_{\lambda}}, \frac{\partial \psi_{n}}{\partial x_{\gamma}}\right)\right],
\end{gathered}
$$

where $a_{\lambda, \gamma}^{\alpha, \beta}, b_{\lambda, \gamma}^{\alpha, \beta}$ and $c_{\lambda, \gamma}^{\alpha, \beta}$ are equal to +1 or -1 , and $\mathscr{P}=\{$ permutations of $(0,1,2,3)\}$.

Now we multiply this equality by $\varphi^{4}$ and we integrate it over $\mathbb{R}^{3}$. The terms in which $\psi_{n}$ appears at most twice are shown to converge to 0 as $n$ goes to $+\infty$ by using (11)-(13), and the same can be done with the terms of the form

$$
\int_{\mathbb{R}^{3}} \varphi^{4} \operatorname{det}\left(\frac{\partial \phi}{\partial x_{\alpha}}, \frac{\partial \psi_{n}}{\partial x_{\beta}}, \frac{\partial \psi_{n}}{\partial x_{\lambda}}, \frac{\partial \psi_{n}}{\partial x_{\gamma}}\right) d x
$$

where $\alpha \neq 0$. Thus the only difficult term is $\int_{\mathbb{R}^{4}} \varphi^{4} \operatorname{det}\left(\phi, \nabla \psi_{n}\right) d x$. 
After some tedious computations one shows that since $\varphi \in \mathscr{D}\left(\mathbb{R}^{3}, \mathbb{R}\right)$, we have

$$
\begin{aligned}
& 3 \int_{\mathbb{R}^{3}} \varphi^{4} \operatorname{det}\left(\phi, \nabla \psi_{n}\right) d x=\int_{\mathbb{R}^{3}} \varphi^{4}\left[\operatorname{det}\left(\psi_{n}, \frac{\partial \phi}{\partial x_{1}}, \frac{\partial \psi_{n}}{\partial x_{2}}, \frac{\partial \psi_{n}}{\partial x_{3}}\right)\right. \\
& +\operatorname{det}\left(\psi_{n}, \frac{\partial \psi_{n}}{\partial x_{1}}, \frac{\partial \phi}{\partial x_{2}}, \frac{\partial \psi_{n}}{\partial x_{3}}\right) \\
& \left.+\operatorname{det}\left(\psi_{n}, \frac{\partial \psi_{n}}{\partial x_{1}}, \frac{\partial \psi_{n}}{\partial x_{2}}, \frac{\partial \phi}{\partial x_{3}}\right)\right] d x \\
& +\int_{\mathbb{R}^{3}}\left(\sum_{(\alpha, \beta, \lambda, \gamma) \in \mathscr{P}} l_{\alpha \beta \lambda \gamma} \operatorname{det}\left(\frac{\partial \phi}{\partial x_{\alpha}}, \frac{\partial \phi}{\partial x_{\beta}}, \frac{\partial \psi_{n}}{\partial x_{\lambda}}, \frac{\partial \psi_{n}}{\partial x_{\gamma}}\right)\right) d x,
\end{aligned}
$$

where the $l_{\alpha \beta \lambda \gamma}$ are integers independent of $\phi$. Then, it is easy to conclude.

Then, once we have proved (26)-(27), we follow the general lines of the proof of Lemma I.1 in [L2].

We are going now to finish the proof of Theorem 1. By using propositions 11, 12, 13 we have shown that either $d(\phi)=1$ or there must be at least $a v_{i} \neq 0$ (and therefore, $\mu_{i} \neq 0$ as well). But if this were to happen, it would mean that the area covered by $\phi_{n}$ in $B\left(x_{i}, r\right)$ with $r$ small is bounded away from zero. And this is in contradiction with Lemma 10, which tells us that the total energy being bounded, the area covered by $\phi_{n}$ in $B\left(x_{i}, r\right)$ is bounded by a constant times $r^{3 / 4}$.

Therefore, $v_{i}=0$ for all $i$ and $d(\phi)=1$, which means that $\phi$ is a minimum for $I_{1}$.

\section{Proof of Theorem 2}

We observe that Theorem 2 is not an existence result, but only gives a sufficient condition for minima of $I_{k}, k \neq 0, \pm 1$, to exist and to be stable in some vague sense. This is due to the fact that we do not know of the relationships existing between the values of $I_{k}$ and $I_{1}$. It seems possible, for instance, that $I_{k}=|k| I_{1}$ for all $k$. In this case, the condition given in Theorem 2 would not be fulfilled. However, it would still be possible that minima of $I_{k}$ exist, but being unstable in some sense.

Proof of Theorem 2. Everything we did in the proof of Theorem 1 applies here except for the ruling out of the dichotomy. Let us then prove that this cannot happen under the conditions of Theorem 2, and the proof will be complete.

We will assume that (18) is satisfied. Then we use Appendix 2 to build up two functions of $X, \phi_{n}^{1}$ and $\phi_{n}^{2}$ such that:

$$
\phi_{n}^{1} \equiv \phi_{n} \text { in } B\left(y_{n}, R\right), \quad \phi_{n}^{2} \equiv \phi_{n} \text { in } B\left(y_{n}, R_{n}\right)^{c} .
$$

As in the proof of Proposition 12 we observe that for $n$ large and $\varepsilon$ small we must have $d\left(\phi_{n}^{1}\right)+d\left(\phi_{n}^{2}\right)=k$ and $\left|d\left(\phi_{n}^{1}\right)\right|+\left|d\left(\phi_{n}^{2}\right)\right|>k$, since the opposite would contradict $I_{k}$ 's definition.

Next we observe that if $(2)$ holds for a fixed $k$, then there exists a constant $m \in(0,1)$ such that $I_{k}<m\left(I_{l}+I_{k-l}\right)$ for all $l \in \mathbb{Z}-\{0, k\}$. Indeed by Proposition 8 and Lemma 9 
we see that if $|l| \geqq 2 k$, then,

$$
I_{1}+I_{k-1} \geqq \frac{6 \gamma \kappa}{4 \pi^{2}}\left|S^{3}\right|(|l|+|k-l|),
$$

and

$$
I_{k} \leqq|k| I_{1}
$$

Moreover, in [E1] we obtained an estimate for $I_{1}$ as follows:

$$
I_{1} \leqq \frac{6 \gamma \kappa}{4 \pi^{2}}\left|S^{3}\right| \sqrt{2}
$$

Hence, for $|l| \geqq 2 k$, we have

$$
\frac{I_{k}}{I_{l}+I_{k-l}} \leqq \frac{\sqrt{2}}{2}
$$

Then we may define $m=\max _{l \in \mathbb{Z}-\{0, k\}}\left(\frac{I_{k}}{I_{l}+I_{k-l}}\right)$ and $m$ satisfies $\frac{\sqrt{2}}{2}<m<1$.

We apply this to $l_{n}=d\left(\phi_{n}^{1}\right)$ and $k_{n}=k-l_{n}=d\left(\phi_{n}^{2}\right)$ to obtain

$$
I_{l_{n}}+I_{k-l_{n}} \leqq \varepsilon+(1+\delta(L+1)) I_{k} .
$$

Therefore if we choose $\varepsilon$ small, $n$ large and $\delta$ such that $1+\delta(L+1)<1 / m$, we reach a contradiction. And this concludes the proof.

Corollary 14. The best constant of the inequality (5) is achieved in $X$. That is, there exists $\bar{\phi}$ in $X$ such that:

$$
\mathscr{E}(\bar{\phi})|d(\bar{\phi})|^{-3 / 4}=\inf \left\{\mathscr{E}(\phi)|d(\phi)|^{-3 / 4} \mid \phi \in X\right\} .
$$

Moreover $\bar{\phi} \in X_{1} \cup X_{2} \cup X_{3}$ and if $\bar{\phi} \in X_{i}$, then $C^{-1}=\left(2 \pi^{2} / \kappa \gamma\right) I_{i} i^{-3 / 4}$.

Proof. By Proposition 8, Lemma 9 and (28) we immediately see that the $\inf \left\{\mathscr{E}(\phi)|d(\phi)|^{-3 / 4} \mid \phi \in X\right\}$ is in the set $\left\{I_{1}, I_{2} 2^{-3 / 4}, I_{3} 3^{-3 / 4}\right\}$.

Now, if this infimum is equal to $I_{1}$ the proof is complete. Assume then this infimum (actually, minimum) is either equal to $I_{2} 2^{-3 / 4}$ or to $I_{3} 3^{-3 / 4}$. In the first case we have $I_{2} 2^{-3 / 4} \leqq I_{1}$, and therefore, $I_{2}<2 I_{1}$. So we apply Theorem 2 to conclude.

Finally, if $I_{3} 3^{-3 / 4} \leqq I_{1}, I_{2} 2^{-3 / 4}$, then,

$$
I_{2}+I_{1} \geqq I_{3} 3^{-3 / 4}\left(1+2^{3 / 4}\right)>I_{3},
$$

and again Theorem 2 allows us to conclude. Indeed, for $k \notin\{0,1,2,3\}$ we use Proposition 8, Lemma 9 and (28) to prove the inequality $I_{k}+I_{3-k}>I_{3}$.

The second case can be treated in a similar way.

We will prove next our last result, i.e. the one given after the statement of Theorem 2, which gives a hint of how Theorem 1 may be applied to the understanding of the structure of static configurations of mesons. As we already said in the introduction, Skyrme was interested in configurations of minimal energy. Moreover, the mesons will correspond to points of high energy in a field of weak energy. 
Let $\phi$ (respectively $\psi$ ) be a minimum of $I_{1}$ (respectively $I_{-1}$ ) such that $\|\phi-P\|_{L^{6}\left(\mathbb{R}^{3}\right)},\|\psi-P\|_{L^{6}\left(\mathbb{R}^{3}\right)}<+\infty$.

The sequence $\left\{\phi_{n}\right\}$ will be constructed as follows:

(a) $\forall x \notin \bigcup_{i=1}^{m} \bar{B}\left(\bar{x}_{i}, \frac{1}{n}\right), \quad \phi_{n}(x)=P$.

(b) if $a_{i}=1$ and $x \in B\left(\bar{x}_{i}, 1 / 8 n\right)$, $\phi_{n}(x)=\phi\left(8 n^{2}\left(x-\bar{x}_{i}\right)\right)$.

(c) If $a_{i}=-1$ and $x \in B\left(\bar{x}_{i}, 1 / 8 n\right)$, $\phi_{n}(x)=\psi\left(8 n^{2}\left(x-\bar{x}_{i}\right)\right)$.

And in $\bigcup_{i=1}^{m}\left(B\left(\bar{x}_{i}, 1 / n\right)-B\left(\bar{x}_{i}, 1 / 8 n\right)\right)$, we define $\phi_{n}$ by using the cutting method introduced in Appendix 2. Let us verify that the sequence $\left\{\phi_{n}\right\}$ satisfies conditions (i)-(iv). (ii) is immediate. (i) and (iv) are easily verified since the degree is a function with integer values. Indeed, since $\mathscr{E}\left(\phi_{n} ; \bigcup_{i=1}^{m}\left(B\left(\bar{x}_{i}, 1 / n\right)-B\left(\bar{x}_{i}, 1 / 8 n\right)\right)\right.$ is small for $n$ large (see Appendix 2) and $|\operatorname{det}(\phi, \nabla \phi)| \leqq 2\left(|\nabla \phi|^{2}+|A(\phi)|^{2}\right)$ a.e., (i) and (iv) must hold. That (iii) holds is a consequence of (6) and Lemma 5. Finally, the last statement follows from Proposition 13.

\section{Appendix 1}

We will give here the expression of the degree, the area and the energy of any function $\phi$ of $X$ in spherical coordinates.

We choose a system of four orthonormal vectors in $\mathbb{R}^{4}$ and we may write any point of $\mathbb{R}^{4}$ with respect to them as:

$$
\begin{aligned}
y= & (\cos \varphi(y) \cos \xi(y) \cos \theta(y), \cos \varphi(y) \cos \xi(y) \sin \theta(y), \\
& \cos \varphi(y) \sin \xi(y), \sin \varphi(y))
\end{aligned}
$$

where $\varphi, \xi \in\left[-\frac{\pi}{2}, \frac{\pi}{2}\right]$ and $\theta \in[0,2 \pi]$.

In the same way, for any $\phi$ in $X$ there are three associated functions defined in $\mathbb{R}^{3}$, $\varphi, \xi$ and $\theta$ satisfying

$$
\begin{aligned}
\phi^{1} & \equiv \cos \varphi \cos \xi \cos \theta, \\
\phi^{2} & \equiv \cos \varphi \cos \xi \sin \theta, \\
\phi^{3} & \equiv \cos \varphi \sin \xi \\
\phi^{4} & \equiv \sin \varphi .
\end{aligned}
$$

As we saw in [E2], the degree of $\phi$ is given by

$$
\frac{1}{2 \pi^{2}} \int_{\mathbb{R}^{3}} \operatorname{det}(\phi, \nabla \phi) d x
$$

and the generalized area covered by $\phi\left(\mathbb{R}^{3}\right)$ by

$$
\int_{\mathbb{R}^{3}}\left|\frac{\partial \phi}{\partial x_{1}} \wedge \frac{\partial \phi}{\partial x_{2}} \wedge \frac{\partial \phi}{\partial x_{3}}\right| d x
$$


Let

$$
\bar{i}=\left(\begin{array}{l}
1 \\
0 \\
0 \\
0
\end{array}\right), \quad \bar{j}=\left(\begin{array}{l}
0 \\
1 \\
0 \\
0
\end{array}\right), \quad k=\left(\begin{array}{l}
0 \\
0 \\
1 \\
0
\end{array}\right) \text { and } \tau=\left(\begin{array}{l}
0 \\
0 \\
0 \\
1
\end{array}\right)
$$

Then, after some lengthy computations we obtain:

$$
\begin{aligned}
\frac{\partial \phi}{\partial x_{1}} \wedge \frac{\partial \phi}{\partial x_{2}} \wedge \frac{\partial \phi}{\partial x_{3}}= & D\left(\cos ^{3} \varphi \cos ^{2} \xi \cos \theta \bar{i}\right. \\
& +\cos ^{3} \varphi \cos ^{2} \xi \sin \theta \bar{j}+\cos ^{3} \varphi \sin \xi \cos \xi \bar{k} \\
& \left.+\sin \varphi \cos ^{2} \varphi \cos \xi \bar{l}\right), \text { with } D=\operatorname{det}(\nabla \theta, \nabla \xi, \nabla \varphi) .
\end{aligned}
$$

Then, $\left|\frac{\partial \phi}{\partial x_{1}} \wedge \cdots \wedge \frac{\partial \phi}{\partial x_{3}}\right|=|D|\left(\cos ^{2} \varphi \cos \xi\right)$ and $\operatorname{det}(\phi, \nabla \phi)=\left(\cos ^{2} \varphi \cos \xi\right) D$. So, for all $\phi$ in $X$ we have

$$
\begin{aligned}
d(\phi) & =\frac{1}{2 \pi^{2}} \int_{\mathbb{R}^{3}} \cos ^{2} \varphi \cos \xi(\nabla \theta \cdot \nabla \xi \wedge \nabla \varphi) d x, \\
\operatorname{Ar}(\phi) & =\int_{\mathbb{R}^{3}} \cos ^{2} \varphi \cos \xi|\nabla \theta \cdot \nabla \xi \wedge \nabla \varphi| d x .
\end{aligned}
$$

Let us now calculate the energy $\mathscr{E}$ in spherical coordinates. Assuming that $\phi$ is written as in (A1) we obtain after some tedious computations

$$
|\nabla \phi|^{2}=|\nabla \varphi|^{2}+\cos ^{2} \varphi|\nabla \xi|^{2}+\cos ^{2} \varphi \cos ^{2} \xi|\nabla \theta|^{2} .
$$

Finally let $\alpha, \beta$ be in $\{1,2,3\}$. For any two functions $f, g$, we set $A_{\alpha, \beta}^{f, g}=$ $\partial f / \partial x_{\alpha} \wedge \partial g / \partial x_{\beta}$, and we obtain

$$
\left|\frac{\partial \phi}{\partial x_{\alpha}} \wedge \frac{\partial \phi}{\partial x_{\beta}}\right|^{2}=\left(\cos ^{2} \varphi\right)\left(A_{\alpha \beta}^{\varphi, \xi}\right)^{2}+\left(\cos ^{2} \varphi \cos ^{2} \xi\right)\left(A_{\alpha \beta}^{\varphi, \theta}\right)^{2}+\left(\cos ^{4} \varphi \cos ^{2} \xi\right)\left(A_{\alpha \beta}^{\xi, \theta}\right)^{2} .
$$

We may then write

$$
|A(\phi)|^{2}=\sum_{\alpha, \beta=1}^{3}\left[\left(\cos ^{2} \varphi\right)\left(A_{\alpha \beta}^{\varphi, \xi}\right)^{2}+\left(\cos ^{2} \varphi \cos ^{2} \xi\right)\left(A_{\alpha \beta}^{\varphi, \theta}\right)^{2}+\left(\cos ^{4} \varphi \cos ^{2} \xi\right)\left(A_{\alpha \beta}^{\xi, \theta}\right)^{2}\right] .
$$

\section{Appendix 2}

In the following we will cut a function $\phi$ of $X$ to make it constant near infinity by the use of a certain family of cut-off functions. We are interested in knowing how much energy we spend doing so.

We will cut as well the functions of $X$ in order to make them constant in the neighborhood of a given point of $\mathbb{R}^{3}$.

Let us introduce a cut-off function $m \in C^{\infty}\left(\mathbb{R}^{3}, \mathbb{R}^{+}\right)$satisfying

$$
0 \leqq m \leqq 1, \quad m \equiv 1 \text { in } B_{1}, \quad m \equiv 0 \text { in } B_{2}^{c} .
$$


Then, for all $R>0$ we define $m^{R}$ by

$$
m^{R}(\cdot)=m\left(\frac{\cdot}{R}\right) .
$$

$m^{R}$ satisfies: $0 \leqq m^{R} \leqq 1, m^{R} \equiv 1$ in $B_{R}, m^{R} \equiv 0$ in $B_{2 R}^{c}$.

Given any function $\phi$ in $X$, we consider its spherical coordinates, as in (A1), and then define a new function $\tilde{\phi} \equiv(\tilde{\varphi}, \tilde{\xi}, \tilde{\theta})$ by

$$
\begin{gathered}
\tilde{\varphi} \equiv\left\{\begin{array}{rl}
\varphi, & |x| \leqq 4 R \\
m_{4 R} \varphi, & |x| \geqq 4 R
\end{array},\right. \\
\tilde{\xi} \equiv\left\{\begin{array}{rl}
\xi, & |x| \leqq 2 R \\
m_{2 R} \xi, & |x| \geqq 2 R
\end{array},\right. \\
\tilde{\theta} \equiv\left\{\begin{aligned}
\theta, & |x| \leqq R \\
m_{R} \theta, & |x| \geqq R .
\end{aligned}\right.
\end{gathered}
$$

We note that $\tilde{\phi} \equiv \phi$ in $B_{R}$ and $\tilde{\phi}(x)=\phi(+\infty)=(1,0,0,0)$ for all $x$ with $|x| \geqq 8 R$. Then we prove the following:

Proposition A2. If $\phi$ is any function of $X$ and we define $\tilde{\phi}$ as above, the relationship between the energies of $\phi$ and $\tilde{\phi}$ is given by the following:

$$
\begin{aligned}
\mathscr{E}\left(\tilde{\phi} ; B_{R}\right) & =\mathscr{E}\left(\phi ; B_{R}\right), \\
\mathscr{E}\left(\tilde{\phi} ; B_{8 R}-B_{R}\right) & =\mathscr{E}\left(\tilde{\phi} ; B_{R}^{c}\right) \leqq C \mathscr{E}\left(\phi ; B_{8 R}-B_{R}\right) .
\end{aligned}
$$

Moreover, $\tilde{\phi}$ is an element of $X$.

Proof. (A9) is immediate. Then let us prove (A10). The first equality in (A10) is also trivial because $\bar{\phi}$ is constant in $B_{8 R}^{c}$. Moreover,

$$
\left.\int_{B_{2 R}-B_{R}}|\nabla \tilde{\phi}|^{2} d x=\int_{B_{2 R^{-}} B_{R}}|\nabla \varphi|^{2}+\cos ^{2} \varphi|\nabla \xi|^{2}+\cos ^{2} \varphi \cos ^{2} \xi\left|\nabla\left(m_{R} \theta\right)\right|^{2}\right) d x .
$$

By using Hölder's inequality and $\left\|m_{R}\right\|_{L^{x}}=1$, we obtain

$$
\begin{aligned}
& \int_{B_{2 R}-B_{R}}|\nabla \tilde{\phi}|^{2} d x \\
& \leqq 2 \int_{B_{2 R}-B_{R}}|\nabla \phi|^{2} d x+2\left(\int_{B_{2 R}-B_{R}}\left|\nabla m_{R}\right|^{3} d x\right)^{2 / 3}\left(\int_{B_{2 R}-B_{R}} \cos ^{2} \varphi \cos ^{2} \xi \theta^{6} d x\right)^{1 / 3} \\
& \left.\leqq 2 \int_{B_{2 R^{-}-B_{R}}}|\nabla \phi|^{2} d x+2 \int_{B_{2}-B_{1}}|\nabla m|^{3} d x\right)^{2 / 3}\left(\int_{B_{2 R}-B_{R}}|\nabla \phi|^{2}\right) \leqq C \int_{B_{2 R}-B_{R}}|\nabla \phi|^{2} .
\end{aligned}
$$

Since

$$
\int_{\mathbb{R}^{3}}\left|\nabla m_{R}\right|^{3} d x=\int_{B_{2 R^{-}-B_{R}}}\left|\nabla m_{R}\right|^{3} d x=\int_{B_{2}-B_{1}}|\nabla m|^{3} d x=\int_{\mathbb{R}^{3}}|\nabla m|^{3} d x \leqq C .
$$

In the other term of the energy $\mathscr{E}$, i.e., $\int_{B_{2 R}-B_{R}}|A(\tilde{\phi})|^{2} d x$, the only integrals to be 
checked are:

and

$$
\int_{B_{2 R}-B_{R}} \cos ^{2} \tilde{\varphi} \cos ^{2} \tilde{\xi}\left[\sum_{\alpha, \tilde{\beta}=1}^{3}\left(A_{\alpha, \beta}^{\tilde{\varphi}, \tilde{\theta}}\right)^{2}\right] d x
$$

$$
\int_{B_{2 R}-B_{R}} \cos ^{4} \tilde{\varphi} \cos ^{2} \xi\left(\sum_{\alpha, \beta=1}^{3}\left(A_{\alpha \beta}^{\xi, \tilde{\sigma}}\right)^{2}\right) d x .
$$

Let us check one of them, for example:

$$
\begin{aligned}
& \int_{B_{2 R}-B_{R}} \cos ^{2} \tilde{\varphi} \cos ^{2} \xi\left(A_{12}^{\tilde{\varphi} \tilde{\theta}}\right)^{2} d x \\
& =\int_{B_{2 R}-B_{R}} \cos ^{2} \varphi \cos ^{2} \xi\left(\frac{\partial \varphi}{\partial x_{1}} \frac{\partial\left(m_{R} \theta\right)}{\partial x_{2}}-\frac{\partial \varphi}{\partial x_{2}} \frac{\partial\left(m_{R} \theta\right)}{\partial x_{1}}\right)^{2} \\
& \leqq 2 \int_{B_{2 R}-B_{R}} \cos ^{2} \varphi \cos ^{2} \xi\left(A_{\alpha \beta}^{\varphi \theta}\right)^{2} d x \\
& \quad+2 \int_{B_{2 R}-B_{R}}\left(\cos ^{2} \varphi \cos ^{2} \xi\right) \theta^{2}\left(\frac{\partial \varphi}{\partial x_{1}} \frac{\partial m_{R}}{\partial x_{2}}-\frac{\partial \varphi}{\partial x_{2}} \frac{\partial m_{R}}{\partial x_{1}}\right)^{2} d x
\end{aligned}
$$

and by using Hölder's inequality, we may estimate the last integral by

$$
\frac{32 \pi^{2}}{R^{2}} \int_{B_{2 R^{-}-B_{R}}}|\nabla \varphi|^{2} d x
$$

since $\theta(x)$ is bounded by $2 \pi$ and

$$
\left\|\nabla m_{R}\right\|_{L^{\infty}\left(\mathbb{R}^{3}\right)}=\frac{1}{R}\|\nabla m\|_{L^{\infty}\left(\mathbb{R}^{3}\right)}=\frac{C}{R} .
$$

In the set $B_{4 R}-B_{2 R}$ we have the following:

$$
\begin{aligned}
\mathscr{E}\left(\tilde{\phi} ; B_{4 R}-B_{2 R}\right)= & \int_{B_{4 R}-B_{2 R}}\left(|\nabla \varphi|^{2}+\cos ^{2} \varphi\left|\nabla\left(m_{2 R} \xi\right)\right|^{2}\right) d x \\
& +\int_{B_{4 R}-B_{2 R}} \cos ^{2} \varphi \sum_{\alpha, \beta}\left(A_{\alpha \beta}^{\varphi, m_{2 R} \xi}\right)^{2} d x
\end{aligned}
$$

since the terms in $\nabla \tilde{\theta}$ vanish. The remaining terms may be estimated as before.

Finally, the last integral to estimate is

$$
\mathscr{E}\left(\tilde{\phi} ; B_{8 R}-B_{4 R}\right)=\int_{B_{8 R}-B_{4 R}}\left|\nabla\left(m_{4 R} \varphi\right)\right|^{2} d x \leqq C \int_{B_{8 R}-B_{4 R}}|\nabla \varphi|^{2} d x .
$$

Remark. If instead of $m$ we consider a new cut-off function $n \in C^{\infty}\left(\mathbb{R}^{3}\right)$ satisfying

$$
0 \leqq n \leqq 1, \quad n \equiv 0 \text { in } B_{1}, \quad n \equiv 1 \text { in } B_{2}^{c},
$$

we could as well "cut" any function $\phi$ of $X$ to obtain $\bar{\phi}$, such that

$$
\begin{aligned}
& \tilde{\phi} \equiv \phi \text { in } B_{R}^{c}, \\
& \tilde{\phi} \equiv “ \phi(0) " \text { in } B_{R / 8}, \\
& \mathscr{E}\left(\tilde{\phi} ; B_{R}-B_{R / 8}\right) \leqq C \mathscr{E}\left(\phi ; B_{R}-B_{R / 8}\right),
\end{aligned}
$$


where $C$ is independent of $\phi$ and $R$, and by $\phi(0)$ we mean the $\lim _{R \rightarrow 0} \frac{1}{\left|B_{R}\right|} \int_{B_{R}} \phi(x) d x$.

To show the last statement we consider two possible cases. If $\phi \in C^{1}\left(\mathbb{R}^{3}, S^{3}\right)$, we can conclude. If $\phi \notin C^{1}\left(\mathbb{R}^{3}, S^{3}\right)$, then by the definition of $X$ there must exist a sequence $\left\{\phi_{n}\right\} \subset C^{1}\left(\mathbb{R}^{3}, S^{3}\right)$ such that $\nabla \phi_{n} \underset{n \rightarrow+\infty}{\longrightarrow} \nabla \phi, A\left(\phi_{n}\right) \underset{n \rightarrow+\infty}{\longrightarrow} A(\phi)$ in $L^{2}\left(\mathbb{R}^{3}, \mathbb{R}^{4}\right)$. Then by using the same arguments as above, we see that $\left\{\tilde{\phi}_{n}\right\} \subset C^{1}\left(\mathbb{R}^{3}, S^{3}\right)$ and

$$
\nabla \tilde{\phi}_{n} \underset{n \rightarrow+\infty}{\longrightarrow} \nabla \tilde{\phi}, \quad A\left(\tilde{\phi}_{n}\right) \underset{n \rightarrow+\infty}{\longrightarrow} A(\tilde{\phi}) \text { in } L^{2}\left(\mathbb{R}^{3}, S^{3}\right)
$$

Hence $\tilde{\phi} \in X$.

\section{References}

[A] Adkins, G. S. Nappi, C. R., Witten, E.: Static properties of nucleons in the Skyrme model. Nucl. Phys. B228, 552-566 (1983)

[B] Ball, J.: Convexity conditions and existence theorems in nonlinear elasticity. Arch. Ration. Mech. Anal. 63, 337-403 (1977)

[B-C] Brézis, H., Coron, J. M.: Large solutions for Harmonic maps in $R^{2}$. Commun. Math. Phys. 92, 203-215 (1983)

[E1] Esteban, M. J.: An isoperimetric inequality in $R^{3}$. (To appear)

[E2] Esteban, M. J.: Existence of symmetric solutions for the Skyrme's problem. (To appear)

[t'H1] t'Hooft, G.: A planar diagram theory for strong interactions. Nucl. Phys. B72, 461-473 (1974)

[t'H2] t'Hooft, G.: A two-dimensional model for mesons. Nucl. Phys. B75, 461-470 (1974)

[K-L] Kapitanski, L. B., Ladyzenskaia, O. A.: On the Coleman's principle concerning the stationary points of invariant functionals. Zap. Nauchn. Semin, LOMI 127, 84-102 (1983)

[LE] Levy, P.: Theorie de l'addition des variables aléatoires. Paris: Gauthier-Villars 1954

[L1] Lions, P. L.: The Concentration-compactness principle in the calculus of variations: Part I.Ann. IHP. Anal. Non Lin. 1, 109-145 (1984); Part II.-Ann. IHP. Anal. Non Lin. 1, 223-283 (1984)

[L2] Lions, P. L.: The Concentration-compactness principle in the calculus of variations. The limit case. Parts I and II: Part I. - Rev. Mat. Iber. I, 1 145-200 (1985); Part II.-Rev. Mat. Iber. 1, 2, 45-121 (1985)

[M] Murat, F.: Compacité par compensation: Conditions nécessaires et suffisantes de continuité faible sous une hypothèse de rang constant. Ann. Soc. Norm. Super. Pisa, IV, vol. VIII, 1, 69$102(1981)$

[N] Nirenberg, L.: Topics in nonlinear functional analysis, N.Y.U. Lectures Notes, 1973-1974

[R] Reshetnyak, Y. G.: Stability theorems for mappings with bounded excursions. Sib. Math. J. 9, 499-512 (1968)

[S1] Skyrme, T. H. R.: A non-linear theory of strong interactions. Proc. Roy. Soc. A247, 260-278 (1958)

[S2] Skyrme, T. H. R.: A unifical model of $K$ and $\Pi$-mesons. Proc. Roy. Soc. A252, 236-245 (1951)

[S3] Skyrme, T. H. R.: A non-lineal field theory. Proc. Roy. Soc. A260, 127-138 (1961)

[TAR] Tartar, L.: Compensated compactness and applications to partial differential equations. Non-linear analysis and mechanics: Heriot-Watt symposium, Vol. IV, Knops, R. J., (ed.), New York: Pitmann 1979

[T1] Taubes, C. H.: Monopoles and maps from $S^{2}$ to $S^{2}$; the topology of the configuration space. Commun. Math. Phys. 95, 345-391 (1984)

[T2] Taubes, C. H.: Min-Max theory for the Yang-Mills-Higgs equations. Commun. Math. Phys. 97, 473-540 (1985)

[U1] Uhlenbeck, K. K.: Removeable singularities in Yang-Mills fields. Commun. Math. Phys. 83, $11-29(1982)$ 
[U2] Uhlenbeck, K. K.: Connections with $L^{p}$ bounds on curvature. Commun. Math. Phys. 83, 31$42(1982)$

[V] Vinh Mau, G. S. Lacombe, M., Loiseau, B., Cottingham, W. N., Lisboa, P.: The static baryonbaryon potential in the SKYRME Model. Preprint

[W] Witten, E.: Baryons in the $1 / N$ expansion. Nucl. Phys. B160, 57-115 (1979)

Communicated by L. Nirenberg

Received December 17, 1985 
